missing. We show that this bias can result in upward confounding. 2) As HSV2 is more infectious than HIV we expect HSV2 to be acquired from coinfected partners first followed by HIV. 3) As coinfection increases HIV viral load HSV2 infection may act as a proxy for a partner's elevated infectiousness with HIV. Both of these mechanisms result in upward bias, the magnitude of which depends on the prevalence of coinfection. 4) Between subject heterogeneity in the risk of disease has been shown to attenuate estimates for any risk factor. We show that this bias can result in significant attenuation of the $\mathrm{HR}$ and that it depends on the prevalence of HIV among subjects' partners and their sexual behaviour. We show that if HIV serodiscordant couples are enrolled all four biases can be removed see Abstract P1-S4.04 Table 1.

Conclusions The standard design is affected by at least four biases that preclude causal interpretations of all such HSV2-HIV studies performed to date. Use of a serodiscordant couple study design can remove these biases. It is impossible to correct previous results as the biases are not all in the same direction and their magnitudes depend on the unknown prevalence and transmissibility of both HSV2 and HIV among partners. These findings are expected to generalise to other STI-HIV risk factor studies and can help inform the decision to test HPV vaccination as an HIV prevention measure.

\section{P1-S4.05 QUANTIFYING SOCIAL DESIRABILITY BIASES IN REPORTED CONDOM USE AMONG FEMALE SEX WORKERS IN SOUTHERN INDIA}

doi:10.1136/sextrans-2011-050108.149

\begin{abstract}
${ }^{1} \mathrm{M}$ Pickles, ${ }^{2} \mathrm{~B} M$ Ramesh, ${ }^{3} \mathrm{R}$ Adhikary, ${ }^{2} \mathrm{~S}$ Rajaram, ${ }^{2} \mathrm{~S}$ Isac, ${ }^{4} \mathrm{R}$ Washington, ${ }^{1} \mathrm{M}$ C Boily, ${ }^{5} \mathrm{~J}$ Bradley, ${ }^{6} \mathrm{~S}$ Moses, ${ }^{7} \mathrm{M}$ Alary. ${ }^{1}$ Imperial College, London, UK; ${ }^{2}$ Karnataka Health Promotion Trust, Bangalore, India; ${ }^{3}$ Family Health International, India; ${ }^{4}$ St John's Research Institute, Bangalore, India; ${ }^{5}$ CHARME-India Project, Bangalore, India; ${ }^{6}$ University of Manitoba, Winnipeg, Canada; ${ }^{7}$ Centre hospitalier affilié universitaire de Québec, Quebec, Canada
\end{abstract}

Background As part of the Avahan HIV initiative in Southern India, surveys were carried out in female sex workers (FSWs) and their clients to quantify condom use. We examine reported condom use measured using different survey methodologies, and consistency of response between FSWs and clients to quantify the effect of social desirability bias.

Methods We use data from 15 districts with IBBA face-to-face interviews (FTFIs) for FSWs and clients. Three of these districts also had special behavioural survey (SBS) FTFIs, informal confidential voting interviews (ICVIs), and polling booth surveys (PBSs) for FSWs. ICVI/ PBS survey methodologies increase anonymity, reducing reporting bias of sensitive questions eg, condom use, and are analysed in more detail. The IBBA and SBS FTFIs differed as blood samples were taken in the IBBA to measure HIV prevalence. We use questions about condom use in last act with regular and occasional FSWs/clients. For the 15 IBBA districts the FSW: client ratio of reported condom use at last act was calculated.

Results In IBBAs, condom use in last act with occasional FSWs reported by clients in the 15 districts is $26 \%$ (range $13-40 \%$ ) lower than FSW condom use with last occasional client, and 28\% (3-46\%) lower for last act with regular FSW/client. In the three districts with extra surveys, from Abstarct P1-S4.05 table 1, FSW reported condom use with occasional clients is broadly comparable, and the maximum difference with the more anonymous ICVI is $5 \%$ with regular clients in Belgaum. On average the IBBA FTFI was only $2 \%$ lower that other methods. Reported condom use with occasional clients was $15-26 \%$ lower than that reported in the IBBA, but the difference between IBBA and PBS was smaller for use with regular clients.
Abstarct P1-S4.05 Table 1 Reported condom use in last act by type of act for FSW surveys in the three districts where all surveys were carried out

\begin{tabular}{|c|c|c|c|c|c|c|}
\hline & \multicolumn{3}{|c|}{$\begin{array}{l}\text { Condom use at last act with } \\
\text { occasional clients/FSWs }\end{array}$} & \multicolumn{3}{|c|}{$\begin{array}{l}\text { Condom use at last act } \\
\text { with regular clients/FSWs }\end{array}$} \\
\hline & $\begin{array}{l}\text { Belgaum } \\
(\%)\end{array}$ & $\begin{array}{l}\text { Bangalore } \\
\text { Urban (\%) }\end{array}$ & $\begin{array}{l}\text { Bellary } \\
(\%)\end{array}$ & $\begin{array}{l}\text { Belgaum } \\
(\%)\end{array}$ & $\begin{array}{l}\text { Bangalore } \\
\text { Urban (\%) }\end{array}$ & $\begin{array}{l}\text { Bellary } \\
(\%)\end{array}$ \\
\hline FSW IBBA R1 FTFI & 96.43 & 93.31 & 82.82 & 86.91 & 75.12 & 77.57 \\
\hline FSW IBBA R2 FTFI & 97.45 & 94.08 & 96.45 & 88.22 & 84.02 & 92.68 \\
\hline FSW SBS FTFI & 91.40 & 85.98 & 90.70 & 82.43 & 91.18 & 94.41 \\
\hline FSW SBS ICVI & 98.18 & 91.53 & 97.67 & 82.39 & 77.43 & 95.78 \\
\hline FSW PBS R3 (May 2007) & 82.75 & 73.33 & 66.78 & 94.94 & 75.68 & 80.21 \\
\hline FSW PBS R4 (0ct 2008) & 88.80 & 79.50 & 80.40 & 88.52 & 87.01 & 80.30 \\
\hline
\end{tabular}

Discussion There is a substantial difference in reported condom use in last act with occasional and regular partner for FSWs and clients. However, the samples of FSW and clients may not be comparable because low-risk clients may be undersampled in hot spots. FSWs report lower condom use with occasional clients in the more anonymous PBS, suggesting this method may reduce social desirability bias more than FTFIs and ICVIs. Based on FSW data only and using a conservative estimate from the largest difference, the FSW IBBA may over-estimate condom use with occasional clients by up to $26 \%$ (mean $17 \%$ ) and with regular clients by $13 \%$ (mean $2 \%$ ).

\section{P1-S4.06 WHAT IMPACT DOES MISSING QUEBEC DATA HAVE ON NATIONAL HIV SURVEILLANCE DATA?}

doi:10.1136/sextrans-2011-050108.150

${ }^{1} \mathrm{~K} L$ Tomas, ${ }^{2} \mathrm{R}$ Bitera, ${ }^{2} \mathrm{M}$ Alary, ${ }^{2} \mathrm{M}$ Fauvel, ${ }^{2} \mathrm{R}$ Parent, ${ }^{2} \mathrm{D}$ Sylvain, ${ }^{2} \mathrm{M}$ Hastie, ${ }^{2} \mathrm{C}$ Claessens, ${ }^{1} \mathrm{~J}$ Halverson, ${ }^{1} \mathrm{C}$ Archibald. ${ }^{1}$ Public Health Agency of Canada, Ottawa, Canada; ${ }^{2}$ nstitut national de sante publique du Quebec, Canada

Objective To quantify the difference in the exposure category breakdowns of national HIV surveillance figures if exposure data from the Institut nationale de Santé Publique du Québec (INSPQ) were included in national datasets.

Background National HIV/AIDS surveillance is coordinated by the Public Health Agency of Canada's (PHAC) Surveillance and Risk Assessment Division's (SRAD). HIV is reportable in all provinces and territories, although the degree of epidemiologic information collected and submitted varies. Quebec's case reports to PHAC come from their laboratory-based surveillance system, which contains positive test reports, by age and sex. All Quebec cases are classified in SRAD's dataset as Not Reported, which contributes to the large proportion of cases at the national level with no known exposure category.

Methods Quebec's provincial HIV surveillance system "Programme de surveillance de l'infection par le VIH au Québec" collects further epidemiological information, including exposure category and risk factor information, although recorded separately from the HIV laboratory test results file. This provincial system's exposure category data was added to existing national surveillance data, and the exposure category breakdowns recalculated, in order to assess change in the proportion of unknown/not reported cases and to quantify the resulting difference in exposure category breakdowns at the national level.

Results With inclusion of Quebec data for 2009, there is a $50 \%$ decrease (from $45.5 \%$ to $23.1 \%$ ) in the proportion of national HIV cases with unknown exposure category. There are also differences in the overall national exposure category breakdowns. For 2009, proportional increases were observed in the men who have sex with men (MSM) and heterosexual-endemic categories $(5.4 \%$ and $2.8 \%$ respectively), while proportional decreases were observed in the exposure categories of injection drug use $(-4.1 \%)$, heterosexual-risk $(-2.0 \%)$, and no-identified-risk heterosexual $(-2.2 \%)$. 